\section{Support Vector Machines for Nonlinear Kernel ARMA System Identification}

Manel Martínez-Ramón, José Luis Rojo-Álvarez, Gustavo Camps-Valls, Jordi Muñoz-Marí, Ángel Navia-Vázquez, Emilio Soria-Olivas, and Aníbal R. Figueiras-Vidal

\begin{abstract}
Nonlinear system identification based on support vector machines (SVM) has been usually addressed by means of the standard SVM regression (SVR), which can be seen as an implicit nonlinear autoregressive and moving average (ARMA) model in some reproducing kernel Hilbert space (RKHS). The proposal of this letter is twofold. First, the explicit consideration of an ARMA model in an RKHS (SVM-ARMA ${ }_{2 K}$ ) is proposed. We show that stating the ARMA equations in an RKHS leads to solving the regularized normal equations in that RKHS, in terms of the autocorrelation and cross correlation of the (nonlinearly) transformed input and output discrete time processes. Second, a general class of SVM-based system identification nonlinear models is presented, based on the use of composite Mercer's kernels. This general class can improve model flexibility by emphasizing the input-output cross information (SVM-ARMA ${ }_{4 K}$ ), which leads to straightforward and natural combinations of implicit and explicit ARMA models (SVR-ARMA ${ }_{2 K}$ and SVR-ARMA ${ }_{4 K}$ ). Capabilities of these different SVM-based system identification schemes are illustrated with two benchmark problems.
\end{abstract}

\section{INTRODUCTION}

A common problem in digital signal processing is to model a functional relationship between two simultaneously recorded discrete-time processes (DTP) [1]. When this relationship is linear and time-invariant, it is usually addressed with autoregressive and moving average (ARMA) modeling, but if linearity cannot be assumed, nonlinear system identificatio techniques are required. General nonlinear models, such as artificia neural networks, wavelet, and fuzzy models, are common and effective choices [1], [2], but the temporal structure of these nonlinear models cannot be easily analyzed, because it remains inside a black-box model.

Support vector machines (SVM) were originally conceived for pattern recognition and classificatio tasks [3], and support vector regression (SVR) was subsequently proposed as the SVM implementation for regression and function approximation [4]. SVMs have been successfully used in a huge variety of problems in signal processing, bioengineering or image processing [5], among others. Main advantage of SVM current algorithms is their capability for giving nonlinear algorithms by the statement of a well-known linear data model (classificatio or regression) in a nonlinearly transformed domain, known as reproducing kernel Hilbert space (RKHS). In [6]-[10], SVR algorithm was used for nonlinear system identification but the time-series

Manuscript received April 20, 2005; revised March 5, 2006. This work was supported in part by Comunidad de Madrid, Spain, under Grant S-0505/TIC/ 0223.

M. Martínez-Ramón, J. L. Rojo-Álvarez, A. Navia-Vázquez, and A. R. Figueiras-Vidal are with Departamento de Teoria de la Señal y Comunicaciones, Universidad Carlos III de Madrid, Madrid 28911, Spain (e-mail: manel@ieee.org; manel@tsc.uc3m.es; jlrojo@tsc.uc3m.es; navia@tsc.uc3m.es; arfv@tsc.uc3m.es).

G. Camps-Valls, J. Muñoz-Marí, and E. Soria-Olivas are with Departament d'Enginyeria Electrònica, Universitat de València, 46100 Burjassot, València, Spain (e-mail: gcamps@uv.es; jordi@uv.es; soriae@uv.es).

Digital Object Identifie 10.1109/TNN.2006.879767 structure of the data was not scrutinized. In [11], SVM was explicitly formulated for modeling linear time-invariant ARMA systems (linear SVM-ARMA), and this kind of formulation has been recently extended to a general framework for linear signal processing problems [12].

This paper introduces an explicit formulation of the ARMA data structure in an RKHS by using the well-known kernel trick. The so-called SVM-ARMA ${ }_{2}$ allows us to study the time-series structure on a straightforward and natural way. Additionally, we introduce a general and still simple class of SVM-based system identificatio algorithms, by using composite kernels. In this context, a second algorithm is presented to take into account the input-output cross information

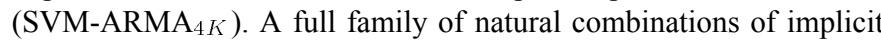
and explicit ARMA models (SVR-ARMA ${ }_{2}$ and SVR-ARMA $4 K$ algorithms) is finall proposed.

The scheme of this work is as follows. Section II summarizes the SVR algorithm for nonlinear system identification Section III presents the novel formulation of an explicit ARMA models in the RKHS. Section IV introduces the use of composite kernels for further model $\mathrm{fl} \mathrm{x}$ ibility. Section V shows the advantages of the proposed methods in some benchmark examples. Section VI gives conclusions and outlines future work. Appendices I and II show the relationships between SVM system identificatio and the classical system identificatio families described in [1]: prediction error models (PEM) and correlation models (CM).

\section{IMPLICIT ARMA SYSTEM IDENTIFICATION WITH SVR}

Previous SVM-based approaches to nonlinear system identificatio have taken advantage of both the kernel trick and the well-developed SVR algorithmic implementations [6]-[10]. The nonlinear SVR-based system identificatio is briefl presented in this section, with the aims of reviewing the kernel trick, highlighting the implicit ARMA nature of this problem statement, and introducing the $\varepsilon$-Huber cost function in this setting.

\section{A. Mercer's Kernels and Nonlinearity}

Let us consider two DTPs, $\left\{u_{n}\right\}$ and $\left\{y_{n}\right\}$, which are the input and the output, respectively, of a nonlinear system. Let $\boldsymbol{y}_{n-1}=$ $\left[y_{n-1}, y_{n-2}, \ldots, y_{n-P}\right]^{T}$ and $\boldsymbol{u}_{n}=\left[u_{n}, u_{n-1}, \ldots, u_{n-Q+1}\right]^{T}$ denote the states of input and output DTP at time instant $n$, so that $\boldsymbol{z}_{n}=\left[\boldsymbol{y}_{n-1}^{T}, \boldsymbol{u}_{n}^{T}\right]^{T}$ is just the vector concatenation of input and output states at that instant. We assume that $P$ and $Q$ are large enough so that the predictable part of the process is completely captured. The SVR-based system identificatio uses a nonlinear transformation $\boldsymbol{\phi}_{z}\left(\boldsymbol{z}_{n}\right): \mathbb{R}^{P} \times \mathbb{R}^{Q} \rightarrow \mathcal{H}_{z}$, which maps the concatenation vector to an RKHS $\mathcal{H}_{z}$, or feature space. For a properly chosen transformation $\boldsymbol{\phi}_{z}$, a linear regression model can be built in $\mathcal{H}_{z}$, and it is given by

$$
y_{n}=\left\langle\boldsymbol{v}, \boldsymbol{\phi}_{z}\left(\boldsymbol{z}_{n}\right)\right\rangle+e_{n}
$$

where $\boldsymbol{v} \in \mathcal{H}_{z},\langle\cdot, \cdot\rangle$ denotes the dot product, and $\left\{e_{n}\right\}$ is a DTP standing for the effect of measurement errors.

In general, the RKHS dimension $\left(H_{z}\right)$ will be greater than the input space dimension $(P+Q)$, and for some choices of the transformation it can be even infinite However, the SVM methodology allows to still work in that high-dimensional RKHS by using Mercer's kernels [13]. If a bivariate function $K_{z}\left(\boldsymbol{z}_{i}, \boldsymbol{z}_{j}\right)$ fulfill Mercer's condition, 
i.e., $\int K_{z}\left(\boldsymbol{z}_{i}, \boldsymbol{z}_{j}\right) f\left(\boldsymbol{z}_{i}\right) f\left(\boldsymbol{z}_{j}\right) \geq 0$ for any square integrable functions $f(\boldsymbol{z})$, then there exist a Hilbert space $\mathcal{H}_{z}$ and a mapping $\phi_{z}$ such that $K_{z}\left(\boldsymbol{z}_{i}, \boldsymbol{z}_{j}\right)=\left\langle\boldsymbol{\phi}_{z}\left(\boldsymbol{z}_{i}\right), \boldsymbol{\phi}_{z}\left(\boldsymbol{z}_{j}\right)\right\rangle$. Therefore, the kernel trick in SVM consists of stating the problem at hand (such as classif cation, regression, and many others) in terms of dot products in the RKHS, and then substituting these products by Mercer's kernels. The kernel expression is actually used in a given SVM algorithm, but neither the mapping function $\phi_{z}$, nor the RKHS, need to be explicitly known.

The widely used Gaussian Mercer's kernel is given by $K_{z}\left(\boldsymbol{z}_{i}, \boldsymbol{z}_{j}\right)=$ $\exp \left(-\left(\left\|\boldsymbol{z}_{i}-\boldsymbol{z}_{j}\right\|^{2} / 2 \sigma^{2}\right)\right)$, where $\sigma$ is the kernel width and can be seen as a parameter that controls the distortion in the input space to provide with an RKHS in which linear regression (1) is an appropriate model. For the Gaussian kernel, the explicit expression of nonlinear transformation $\boldsymbol{\phi}_{z}(\boldsymbol{z})$ is unknown, and the corresponding RKHS dimension is inf nite [13].

\section{B. The $\varepsilon$-Huber Residual Cost}

SVM algorithms minimize a cost function of the residuals (CFR) that is regularized with the $L_{2}$ norm of the model coeff cients in an RKHS. Two CFRs have been used in previous SVM-based system identif cation research: 1) the $\varepsilon$-insensitive CFR [6], [7], which yields sparse solutions, is essentially a $L_{1}$ cost, and hence appropriate for dealing with outliers, and 2) the quadratic CFR used in least-squares (LS) SVM approaches [8]-[10], which is optimal when Gaussian noise is present, but it is sensitive to outliers, and more, it does not produce sparse solutions. The $\varepsilon$-Huber cost [11] contains the preceding ones as particular cases, and it is expressed as

$$
\ell_{P}\left(e_{n}\right)= \begin{cases}0, & \left|e_{n}\right| \leq \varepsilon \\ \frac{1}{2 \gamma}\left(\left|e_{n}\right|-\varepsilon\right)^{2}, & \varepsilon<\left|e_{n}\right| \leq e_{C} \\ C\left(\left|e_{n}\right|-\varepsilon\right)-\frac{1}{2} \gamma C^{2}, & \left|e_{n}\right|>e_{C}\end{cases}
$$

where $e_{c}=\varepsilon+\gamma C$. Parameter $\gamma$ controls the width of the $L_{2}$ interval between $\varepsilon$ and $e_{c}$, so that the function is continuous and derivable, and the $L_{1}$ interval has slope $C$. The $\varepsilon$-insensitivity zone provides with sparse solutions in SVM formulation, which is a very desirable characteristic in nonlinear formulations. The quadratic cost is optimal, in a maximum likelihood (ML) sense, when the noise is Gaussian, whereas the linear cost is optimal for exponential noise. Here, we propose to use the $\varepsilon$-Huber CFR because it has the ability to deal simultaneously with different kinds of noise [11]. The use of $\varepsilon$-insensitive CFR is not appropriate when Gaussian noise is present in the data, whereas a quadratic CFR (according to LS-SVM) does not produce sparse solutions.

\section{Algorithm Statement for SVR System Identification}

The algorithm for SVR system identif cation using the proposed $\varepsilon$-Huber cost reduces to the minimization of

$$
\begin{aligned}
L_{P}^{\mathrm{SVR}}\left(v_{j}, \xi_{n}^{(*)}\right)=\frac{1}{2} \sum_{j=1}^{H_{z}} v_{j}^{2} & +\frac{1}{2 \gamma} \sum_{n \in I_{1}}\left(\xi_{n}^{2}+\xi_{n}^{* 2}\right) \\
& +C \sum_{n \in I_{2}}\left(\xi_{n}+\xi_{n}^{*}\right)-\sum_{n \in I_{2}} \frac{\gamma C^{2}}{2}
\end{aligned}
$$

where $\xi_{n}, \xi_{n}^{*}$, are the slack variables or losses, $I_{1}$ is the set of samples for which $\varepsilon \leq \xi_{n}^{(*)} \leq e_{c}, I_{2}$ is the set of samples for which $\xi_{n}^{(*)}>e_{c}$, and constrained to

$$
\begin{aligned}
& y_{n}-\boldsymbol{v}^{T} \boldsymbol{\phi}_{z}\left(\boldsymbol{z}_{n}\right) \leq \varepsilon+\xi_{n} \\
& \forall n=n_{0}, \ldots, N \\
& -y_{n}+\boldsymbol{v}^{T} \boldsymbol{\phi}\left(\boldsymbol{z}_{n}\right) \leq \varepsilon+\xi_{n}^{*} \\
& \forall n=n_{0}, \ldots, N
\end{aligned}
$$

where $\xi_{n}^{(*)} \geq 0, n_{0}$ is given by the required initial conditions (without loss of generality, $n_{0}=1$ and null initial conditions), $N$ is the number of available samples, and $\xi_{n}^{(*)}$ denotes both $\xi_{n}$ and $\xi_{n}^{*}$.

The Lagrangian for this problem is obtained by introducing a coeff cient (Lagrange multiplier) for each constraint [14]. In particular, $\alpha_{n}$ and $\alpha_{n}^{*}$ are the (nonnegative) Lagrange multipliers corresponding to (4) and (5), respectively. By making zero the gradient of the Lagrangian with respect to $v_{j}$ and $\xi_{n}^{(*)}$, we obtain

$$
\boldsymbol{v}=\sum_{n=1}^{N}\left(\alpha_{n}-\alpha_{n}^{*}\right) \boldsymbol{\phi}_{z}\left(\boldsymbol{z}_{n}\right)=\sum_{n=1}^{N} \beta_{n} \boldsymbol{\phi}_{z}\left(\boldsymbol{z}_{n}\right)
$$

and $0 \leq \alpha_{n}^{(*)} \leq C$, where $\beta_{n}=\alpha_{n}-\alpha_{n}^{*}$. After introducing these conditions into the Lagrangian, the primal variables are removed, and a term-grouping can be done by writing down the Gram matrix of dot products in the RKHS, or kernel matrix

$$
\boldsymbol{G}_{i j}=\left\langle\boldsymbol{\phi}_{z}\left(\boldsymbol{z}_{i}\right), \boldsymbol{\phi}_{z}\left(\boldsymbol{z}_{j}\right)\right\rangle=K_{z}\left(\boldsymbol{z}_{i}, \boldsymbol{z}_{j}\right)
$$

The dual problem consists in maximizing with constraints

$$
\begin{aligned}
L_{D}^{\mathrm{SVR}}\left(\alpha_{n}^{(*)}\right)=-\frac{1}{2}\left(\boldsymbol{\alpha}-\boldsymbol{\alpha}^{*}\right)^{T} & {[\boldsymbol{G}+\gamma \boldsymbol{I}]\left(\boldsymbol{\alpha}-\boldsymbol{\alpha}^{*}\right) } \\
& +\left(\boldsymbol{\alpha}-\alpha^{*}\right)^{T} \boldsymbol{y}-\varepsilon \mathbf{1}^{T}\left(\boldsymbol{\alpha}+\boldsymbol{\alpha}^{*}\right)
\end{aligned}
$$

where $\boldsymbol{\alpha}^{(*)}=\left[\alpha_{1}^{(*)}, \ldots, \alpha_{N}^{(*)}\right]^{T}$ and $\boldsymbol{y}=\left[y_{1}, \ldots, y_{N}\right]^{T}$. This is a constrained quadratic programming $(\mathrm{QP})$ problem that has a single minimum. It can be shown that the predicted output for a new observed sample $y_{r}$, given $\boldsymbol{z}_{r}$, is

$$
\hat{y}_{r}=\sum_{n=1}^{N} \beta_{n} K_{z}\left(\boldsymbol{z}_{n}, \boldsymbol{z}_{r}\right) .
$$

This solution is expressed in terms of the observation vectors, and hence, if sparsity is allowed in the $\varepsilon$-Huber CFR by making $\varepsilon>0$, only some of the Lagrange multipliers are nonzero. Those samples with a nonzero coeff cient are called support vectors, and they contain all the information that is relevant for building the model.

Property 1: The following nonlinear relationship between the residuals and the model coeff cients holds:

$$
\beta_{n}=g_{n l}\left(e_{n}\right)= \begin{cases}\operatorname{sign}\left(e_{n}\right) C, & \left|e_{n}\right| \geq e_{c} \\ \frac{\operatorname{sign}\left(e_{n}\right)}{\gamma}\left(\left|e_{n}\right|-\varepsilon\right), & \varepsilon \leq\left|e_{n}\right| \leq e_{c} \\ 0, & \left|e_{n}\right|<\varepsilon .\end{cases}
$$

Proof: When using the $\varepsilon$-Huber CFR in SVM regression-like problems, a straightforward relationship between the residuals and the Lagrange multipliers can be derived from the Karush-Khun-Tucker (KKT) conditions [11], [12]. We have that $\alpha_{n}=C$ for $e_{n} \geq e_{c}$, that $\alpha_{n}=(1 / \gamma)\left(e_{n}-\varepsilon\right)$ for $\varepsilon \leq e_{n} \leq e_{c}$, and that $\alpha_{n}=0$ for $e_{n}<\varepsilon$. Also, we have that $\alpha_{n}^{*}=C$ for $e_{n} \leq-e_{c}$, that $\alpha_{n}^{*}=(1 / \gamma)\left(-e_{n}-\varepsilon\right)$ for $-\varepsilon \geq e_{n} \geq-e_{c}$, and that $\alpha_{n}^{*}=0$ for $e_{n}>-\varepsilon$. Given that $\beta_{n}=\alpha_{n}-\alpha_{n}^{*}$, then (10) holds.

According to (9), nonlinear relationship (10) can be conveniently used to control the impact of an outlier on the f nal solution by choosing appropriate values of the cost function parameters [12], i.e., an outlier will have, at most, a weight $\left|\beta_{n}\right|=C$. However, $\beta_{n}$ for $\varepsilon<\left|e_{n}\right|<e_{C}$ can be more fexibly valuated than in $\varepsilon$-insensitive CFR. Free parameters of both the CFR and Mercer's kernel are usually determined in SVM algorithms by using cross-validation search. 


\section{EXPLICIT ARMA MODELS IN RKHS}

The formulation presented in Section II uses a time-series model given by a nonlinear regression in an RKHS, whose input space is given by the concatenated vector $\boldsymbol{z}_{n}$. Therefore, that model can be considered as an ARMA model only in a wide and implicit sense, given that both the AR and the MA component of the observed DTP are stacked and jointly transformed into that RKHS. Although this can be a valid and powerful approach, no useful insight about the temporal statistical properties of the data can be gained. This is a similar situation to the neural network (NN)-based analysis of time series, where the temporal structure remains inside a black-box equation [2]. Alternatively, we propose here to build an explicit ARMA model in some given RKHS, by taking advantage of Mercer's kernels, which will allow us to study the time-series structure of the data, even it being a nonlinear model.

Assume that both the input and the output DTP state vectors can be separately mapped to $\mathcal{H}_{u}$ and $\mathcal{H}_{y}$, by using two possibly different nonlinear mappings $\boldsymbol{\phi}_{u}\left(\boldsymbol{u}_{n}\right): \mathbb{R}^{Q} \rightarrow \mathcal{H}_{u}$ and $\boldsymbol{\phi}_{y}\left(\boldsymbol{y}_{n}\right): \mathbb{R}^{P} \rightarrow \mathcal{H}_{y}$, respectively. A linear MA (AR) model component can be built in $\mathcal{H}_{u}$ $\left(\mathcal{H}_{y}\right)$, and now, the ARMA difference equation is

$$
y_{n}=\boldsymbol{a}^{T} \boldsymbol{\phi}_{y}\left(\boldsymbol{y}_{n-1}\right)+\boldsymbol{b}^{T} \boldsymbol{\phi}_{u}\left(\boldsymbol{u}_{n}\right)+e_{n}
$$

where $\boldsymbol{b}=\left[b_{1}, \ldots, b_{H_{u}}\right]^{T}$ and $\boldsymbol{a}=\left[a_{1}, \ldots, a_{H_{y}}\right]^{T}$ are vectors determining the MA and the AR coeff cients of the system, respectively, in the RKHS; and $H_{u}$ and $H_{y}$ are the RKHS dimensions.

The primal problem can be here formulated as the minimization of

$$
\begin{array}{r}
L_{P}^{2 k}\left(a_{i}, b_{j}, \xi_{n}^{(*)}\right)=\frac{1}{2} \sum_{i=1}^{H_{y}} a_{i}^{2}+\sum_{j=1}^{H_{u}} b_{j}^{2}+\frac{1}{2 \gamma} \sum_{n \in I_{1}}\left(\xi_{n}^{2}+\xi_{n}^{* 2}\right) \\
+C \sum_{n \in I_{2}}\left(\xi_{n}+\xi_{n}^{*}\right)-\sum_{n \in I_{2}} \frac{\gamma C^{2}}{2}
\end{array}
$$

constrained to

$$
\begin{array}{r}
y_{n}-\boldsymbol{a}^{T} \boldsymbol{\phi}_{y}\left(\boldsymbol{y}_{n-1}\right)-\boldsymbol{b}^{T} \boldsymbol{\phi}_{u}\left(\boldsymbol{u}_{n}\right) \leq \varepsilon+\xi_{n} \\
-y_{n}+\boldsymbol{a}^{T} \boldsymbol{\phi}_{y}\left(\boldsymbol{y}_{n-1}\right)+\boldsymbol{b}^{T} \boldsymbol{\phi}_{u}\left(\boldsymbol{u}_{n}\right) \leq \varepsilon+\xi_{n}^{*}
\end{array}
$$

and $\xi_{n}^{(*)} \geq 0$, for $n=1, \ldots, N$. By stating the Lagrangian and making its gradient zero, the AR and MA vector coeff cients are given by

$$
\boldsymbol{a}=\sum_{n=1}^{N} \beta_{n} \boldsymbol{\phi}_{y}\left(\boldsymbol{y}_{n-1}\right) \quad \boldsymbol{b}=\sum_{n=1}^{N} \beta_{n} \boldsymbol{\phi}_{u}\left(\boldsymbol{u}_{n}\right)
$$

which is a different expression for the model coeff cients in (6), because AR and MA coeff cients are now uncoupled in the RKHS.

After introducing (15) into the Lagrangian, we can identify two different Gram matrices, one for the input and other for the output DTP, denoted as

$$
\begin{aligned}
& \boldsymbol{R}_{y, i j}=\left\langle\boldsymbol{\phi}_{y}\left(\boldsymbol{y}_{i-1}\right), \boldsymbol{\phi}_{y}\left(\boldsymbol{y}_{j-1}\right)\right\rangle=K_{y}\left(\boldsymbol{y}_{i-1}, \boldsymbol{y}_{j-1}\right) \\
& \boldsymbol{R}_{u, i j}=\left\langle\boldsymbol{\phi}_{u}\left(\boldsymbol{u}_{i}\right), \boldsymbol{\phi}_{u}\left(\boldsymbol{u}_{j}\right)\right\rangle=K_{u}\left(\boldsymbol{u}_{i}, \boldsymbol{u}_{j}\right) .
\end{aligned}
$$

Equations (16) and (17) can be seen as uncoupled Gram matrices that also account for the sample estimators of input and output DTP autocorrelation functions [15], respectively, in the RKHS. The dual problem consists now in the constrained maximization of

$$
\begin{aligned}
L_{D}^{2 k}\left(\alpha_{n}^{(*)}\right)=-\frac{1}{2}\left(\boldsymbol{\alpha}-\boldsymbol{\alpha}^{*}\right)^{T} & {\left[\boldsymbol{R}_{u}+\boldsymbol{R}_{y}+\gamma \boldsymbol{I}\right]\left(\boldsymbol{\alpha}-\boldsymbol{\alpha}^{*}\right) } \\
& +\left(\boldsymbol{\alpha}-\boldsymbol{\alpha}^{*}\right)^{T} \boldsymbol{y}-\varepsilon \mathbf{1}^{T}\left(\boldsymbol{\alpha}+\boldsymbol{\alpha}^{*}\right) .
\end{aligned}
$$

The output for a new observation vector is obtained as

$$
\hat{y}_{r}=\sum_{n=1}^{N} \beta_{n}\left(K_{y}\left(\boldsymbol{y}_{n-1}, \boldsymbol{y}_{r-1}\right)+K_{u}\left(\boldsymbol{u}_{n}, \boldsymbol{u}_{r}\right)\right) .
$$

Note that the model complexity, in terms of number of coeff cients, is, as in (9), equal to the number of training samples $N$. We will denote this algorithm as SVM-ARMA $2 K$.

To gain further insight about the structure that SVM-ARMA $\mathrm{A}_{2}$ has, we can analyze the temporal structure of the proposed model. According to [1], there are two main general classes of system identif cation algorithms: prediction error methods (PEM), which are based on the minimization of a function of the residual variance for a given model (e.g., least-squares and/or maximum a posteriori estimators); and correlation methods (CM), which minimize the cross correlation between a (possibly nonlinear) function of the residuals and some transformation of the data. In [11], a comparison of linear SVM-ARMA system identif cation with PEM and CM was presented. The SVM$\mathrm{ARMA}_{2 K}$ nonlinear system identif cation model solves the regularized normal equations in some RKHS while minimizing the cross correlation between the data and a nonlinear function of the residuals, as shown in Appendices I and II. This nonlinear relationship is determined by the free parameters of the $\varepsilon$-Huber CFR.

\section{SVM SYSTEM IDENTIFICATION WITH COMPOSITE KERNELS}

In Sections II and III, we have described the SVR-based and the SVM-ARMA $_{2}$ system identif cation algorithms. Two questions can be raised at this moment. First, note that (19) in SVM-ARMA ${ }_{2 K}$ shows an apparent uncoupling between the input and the output DTP in the f nal solution, with no explicit consideration of the (maybe relevant) cross information between them. Although we are solving the normal equations in the RKHS, and the cross correlation is indeed implicitly considered therein, the SVM-ARMA $2 K$ model could be somewhat limited in the cases when strong cross information was present. Therefore, we will look for an SVM-ARMA system identif cation model capable of considering a cross comparison between input and output DTP states, when this becomes necessary in the problem at hand. This new algorithm will be called SVM-ARMA $4 K$. Second, if we observe prediction equations (9) and (19), we can think of the possibility of combining them into a joint model for improving performance and f exibility simultaneously. These two additional algorithms are called SVR-ARMA $_{2 K}$ and SVR-ARMA $4 K$.

In this section, we frst describe the elements of a generic nonlinear mapping into an RKHS in an SVM system identif cation problem. Then, we use composite kernels as direct sum of different RKHS (a well-known result of functional analysis theory, see e.g., [16]), which allows us both to represent the previously described SVM models, and to formulate the aforementioned new system identif cation algorithms.

\section{A. Generic SVM Algorithm for System Identification}

Property 2: Let $\boldsymbol{\phi}\left(\boldsymbol{z}_{n}\right)$ be a composite nonlinear transformation (into an RKHS $\mathcal{H}$ ) given by the concatenation of $M$ single nonlinear transformations to their RKHS, i.e.,

$$
\boldsymbol{\phi}\left(\boldsymbol{z}_{n}\right)=\left[\boldsymbol{\phi}_{1}\left(\boldsymbol{z}_{n}\right)^{T}, \boldsymbol{\phi}_{2}\left(\boldsymbol{z}_{n}\right)^{T}, \ldots, \boldsymbol{\phi}_{M}\left(\boldsymbol{z}_{n}\right)^{T}\right]^{T}
$$

The corresponding SVM system identif cation model is given by $y_{n}=\left\langle\boldsymbol{w}, \boldsymbol{\phi}\left(\boldsymbol{z}_{n}\right)\right\rangle+e_{n}$, where $\boldsymbol{w} \in \mathcal{H}$. The kernel matrix is ${ }_{3} \boldsymbol{K}_{i j}=\left\langle\boldsymbol{\phi}\left(\boldsymbol{z}_{i}\right), \boldsymbol{\phi}\left(\boldsymbol{z}_{j}\right)\right\rangle$, and it allows to state a dual QP problem 
that yields the model coeff cients. The dual problem consists of maximizing with constraints

$$
\begin{aligned}
L_{D}\left(\alpha_{n}^{(*)}\right)=-\frac{1}{2}\left(\boldsymbol{\alpha}-\boldsymbol{\alpha}^{*}\right)^{T}[ & \boldsymbol{K}+\gamma \boldsymbol{I}]\left(\boldsymbol{\alpha}-\boldsymbol{\alpha}^{*}\right) \\
& +\left(\boldsymbol{\alpha}-\boldsymbol{\alpha}^{*}\right)^{T} \boldsymbol{y}-\varepsilon \mathbf{1}^{T}\left(\boldsymbol{\alpha}+\boldsymbol{\alpha}^{*}\right)
\end{aligned}
$$

and the prediction model is $\hat{y}_{r}=\sum_{n=1}^{N} \beta_{n} K\left(\boldsymbol{z}_{n}, \boldsymbol{z}_{r}\right)$.

Proof: The derivation is similar to those presented in Sections II and III.

Property 3: VR From Composite Kernels: It is straightforward to see that the SVR system identif cation model is obtained for $\boldsymbol{\phi}\left(\boldsymbol{z}_{n}\right)=$ $\boldsymbol{\phi}_{z}\left(\boldsymbol{z}_{n}\right)$. In this case, the kernel matrix is (7). Given that prediction model is (9), where a single kernel is used, the explicit consideration of input and output DTP is lost, and the normal equations are generated in an RKHS where the input and output effects are coupled.

Property 4: SVM-ARMA $A_{2 K}$ From Composite Kernels: The proposed SVM-ARMA ${ }_{2 K}$ algorithm can be obtained from a composite kernel formulation by using $\boldsymbol{\phi}\left(\boldsymbol{z}_{n}\right)=\left[\boldsymbol{\phi}_{y}\left(\boldsymbol{y}_{n-1}\right)^{T}, \boldsymbol{\phi}_{u}\left(\mathbf{u}_{n}\right)^{T}\right]^{T}$. It is straightforward to see that the model kernel is

$$
\begin{aligned}
K\left(\boldsymbol{z}_{i}, \boldsymbol{z}_{j}\right) & =\left\langle\left[\boldsymbol{\phi}_{y}\left(\boldsymbol{y}_{i-1}\right)^{T}, \boldsymbol{\phi}_{u}\left(\boldsymbol{u}_{i}\right)^{T}\right]^{T},\left[\boldsymbol{\phi}_{y}\left(\boldsymbol{y}_{j-1}\right)^{T}, \boldsymbol{\phi}_{u}\left(\boldsymbol{u}_{j}\right)^{T}\right]^{T}\right\rangle \\
& =K_{y}\left(\boldsymbol{y}_{i-1}, \boldsymbol{y}_{j-1}\right)+K_{u}\left(\boldsymbol{u}_{i}, \boldsymbol{u}_{j}\right)
\end{aligned}
$$

where the kernel (being the sum of two kernels) accounts for the input and output DTP.

\section{B. Composite Kernels for Input-Output Cross Information}

Composite kernels can be used to emphasize, if necessary, the cross information between input and output DTP. Assume a nonlinear mapping $\varphi(\cdot)$ into an RKHS $\mathcal{H}_{\varphi}$ and three linear transformations $\boldsymbol{A}_{i}$ from $\mathcal{H}_{\varphi}$ to $\mathcal{H}_{i}, i=1,2,3$. Note, however, that in this case, $\boldsymbol{u}_{n}$ and $\boldsymbol{y}_{n}$ need to have the same dimension for the formulation to be valid. For simplicity, we force $P^{\prime}=Q^{\prime}=\max (P, Q)$, which yields input and output vectors $\boldsymbol{u}_{n}^{\prime}$ and $\boldsymbol{y}_{n-1}^{\prime}$ that are ensured to contain all the relevant time-series information (plus some amount of redundant information).

For the following composite transformation:

$$
\boldsymbol{\phi}\left(\boldsymbol{z}^{\prime}\right)=\left[\boldsymbol{A}_{1} \varphi\left(\boldsymbol{u}^{\prime}\right)^{T}, \boldsymbol{A}_{2} \boldsymbol{\varphi}\left(\boldsymbol{y}^{\prime}\right)^{T}, \boldsymbol{A}_{3}\left(\boldsymbol{\varphi}\left(\boldsymbol{u}^{\prime}\right)+\boldsymbol{\varphi}\left(\boldsymbol{y}^{\prime}\right)\right)^{T}\right]^{T}
$$

the obtained kernel is

$$
\begin{aligned}
K\left(\boldsymbol{z}_{i}^{\prime}, \boldsymbol{z}_{j}^{\prime}\right)= & \varphi^{T}\left(\boldsymbol{y}_{i}^{\prime}\right) \boldsymbol{R}_{1} \boldsymbol{\varphi}\left(\boldsymbol{y}_{j}^{\prime}\right)+\varphi^{T}\left(\boldsymbol{u}_{i}^{\prime}\right) \boldsymbol{R}_{2} \varphi\left(\boldsymbol{u}_{j}^{\prime}\right) \\
& +\boldsymbol{\varphi}^{T}\left(\boldsymbol{y}_{i-1}^{\prime}\right) \boldsymbol{R}_{3} \varphi\left(\boldsymbol{u}_{j}^{\prime}\right)+\varphi^{T}\left(\boldsymbol{u}_{i}^{\prime}\right) \boldsymbol{R}_{3} \varphi\left(\boldsymbol{y}_{j-1}^{\prime}\right)
\end{aligned}
$$

where $\boldsymbol{R}_{1}=\boldsymbol{A}_{1}^{T} \boldsymbol{A}_{1}+\boldsymbol{A}_{3}^{T} \boldsymbol{A}_{3}, \boldsymbol{R}_{2}=\boldsymbol{A}_{2}^{T} \boldsymbol{A}_{2}+\boldsymbol{A}_{3}^{T} \boldsymbol{A}_{3}$, and $\boldsymbol{R}_{3}=$ $\boldsymbol{A}_{3}^{T} \boldsymbol{A}_{3}$ are three (independent) def nite-positive matrices. The last two terms can be grouped into a Mercer's kernel $K_{u y}$ accounting for cross information

$$
K_{u y}\left(\boldsymbol{u}_{i}^{\prime}, \boldsymbol{y}_{j-1}^{\prime}\right)=K_{3}\left(\boldsymbol{y}_{i-1}^{\prime}, \boldsymbol{u}_{j}^{\prime}\right)+K_{3}\left(\boldsymbol{u}_{i}^{\prime}, \boldsymbol{y}_{j-1}^{\prime}\right)
$$

which is warranted to be a valid Mercer kernel if $K_{3}$ is a valid Mercer kernel. Then, the $\mathrm{f}$ nal kernel expression is

$$
K\left(\boldsymbol{z}_{i}^{\prime}, \boldsymbol{z}_{j}^{\prime}\right)=K_{y}\left(\boldsymbol{y}_{i-1}, \boldsymbol{y}_{j-1}\right)+K_{u}\left(\boldsymbol{u}_{i}, \boldsymbol{u}_{j}\right)+K_{u y}\left(\boldsymbol{u}_{i}^{\prime}, \boldsymbol{y}_{j-1}^{\prime}\right)
$$

TABLE I

SYSTEM IDENTIFICATION SIMULATION RESULTS. BOLD AND ITALICS INDICATE THE Two Best Models FOR EACH MERIT FIGURE

\begin{tabular}{l|c||c|c|c|c|c}
\hline \hline & Eq. & ME & MSE & MAE & $r$ & nMSE \\
\hline \hline SVR & $(9)$ & 0.29 & 3.08 & 1.08 & 0.991 & -0.82 \\
\hline SVM-ARMA $_{2} K$ & $(19)$ & 0.14 & $\mathbf{2 . 0 8}$ & $\mathbf{0 . 8 8}$ & $\mathbf{0 . 9 9 2}$ & $\mathbf{- 0 . 9 2}$ \\
SVR-ARMA $_{2} K$ & $(26)$ & 0.13 & 2.13 & 0.90 & 0.991 & -0.88 \\
SVM-ARMA $_{4} K$ & $(26)$ & 0.16 & 2.50 & 0.95 & 0.991 & -0.85 \\
SVR-ARMA $_{4} K$ & $(30)$ & $\mathbf{0 . 0 3}$ & 3.76 & 1.11 & 0.976 & -0.77 \\
\hline
\end{tabular}

and using this kernel in the generic SVM system identif cation algorithm gives us the so-called SVM-ARMA $4 K$ algorithm. It can be seen that we are now using four different kernels to build the composite kernel. A cross information analysis of this algorithm can be made, according to the corresponding normal equations in the RKHS for this case (not included here and left as further work).

\section{Composite Kernels for Improved Versatility}

Instead of using separately the proposed algorithms for SVM system identif cation, one can think of using in a collaborative way the different kernel structures that have been presented here.

Property 5: The f rst possibility is using three concatenated transformations into RKHS, one for input $\boldsymbol{u}_{n}$, one for output $\boldsymbol{y}_{n-1}$, and one for their concatenation $\boldsymbol{z}_{n}$. The result is a combination between the SVM-ARMA and the SVR structures described in the preceding sections. The transforming concatenation is

$$
\boldsymbol{\phi}\left(\boldsymbol{z}_{n}\right)=\left[\boldsymbol{\phi}_{y}\left(\boldsymbol{y}_{n-1}\right)^{T}, \boldsymbol{\phi}_{u}\left(\boldsymbol{u}_{n}\right)^{T}, \boldsymbol{\phi}_{z}\left(\boldsymbol{z}_{n}\right)^{T}\right]^{T} .
$$

It is straightforward to see that the corresponding kernel is

$$
K\left(\boldsymbol{z}_{i}, \boldsymbol{z}_{j}\right)=K_{y}\left(\boldsymbol{y}_{i-1}, \boldsymbol{y}_{j-1}\right)+K_{u}\left(\boldsymbol{u}_{i}, \boldsymbol{u}_{j}\right)+K_{z}\left(\boldsymbol{z}_{i}, \boldsymbol{z}_{j}\right)
$$

and its introduction in the generic SVM system identif cation model yields the so-called SVR-ARMA ${ }_{2}$ algorithm.

Property 6: A composite transformation given by

$$
\boldsymbol{\phi}\left(\boldsymbol{z}^{\prime}\right)=\left[\boldsymbol{A}_{1} \varphi\left(\boldsymbol{u}^{\prime}\right)^{T}, \boldsymbol{A}_{2} \boldsymbol{\varphi}\left(\boldsymbol{y}^{\prime}\right)^{T}, \boldsymbol{A}_{3}\left(\boldsymbol{\varphi}\left(\boldsymbol{u}^{\prime}\right)+\boldsymbol{\varphi}\left(\boldsymbol{y}^{\prime}\right)\right)^{T}, \boldsymbol{\phi}_{z}(\boldsymbol{z})^{T}\right]^{T}
$$

gives the following composite kernel:

$$
\begin{aligned}
K\left(\boldsymbol{z}_{i}^{\prime}, \boldsymbol{z}_{j}^{\prime}\right)=K_{y}\left(\boldsymbol{y}_{i-1}, \boldsymbol{y}_{j-1}\right) & +K_{u}\left(\boldsymbol{u}_{i}, \boldsymbol{u}_{j}\right) \\
& +K_{u y}\left(\boldsymbol{u}_{i}^{\prime}, \boldsymbol{y}_{j-1}^{\prime}\right)+K_{z}\left(\boldsymbol{z}_{i}, \boldsymbol{z}_{j}\right)
\end{aligned}
$$

which produces the SVR-ARMA $4 K$ algorithm.

Note that SVR-ARMA $K$ and SVR-ARMA $4 K$ have not a straightforward interpretation in terms of normal equations in the RKHS, but rather they can contain all the relevant model information that can be extracted from the data by each component kernel. Therefore, despite that SVM-ARMA and SVR nonlinear system identif cation are different problem statements, both underlying models can be combined and embedded into a more general SVM signal processing framework for nonlinear system identif cation.

In conclusion, we can say that composite kernels can be used to provide us with model f exibility in terms of: 1) emphasized consideration, if necessary, of the input-output cross information; and 2) straightfor4 ward and natural combinations of implicit and explicit ARMA models. 
TABLE II

NMSE IN VALIDATION SET FOR THE SVM ModELS AND METHODS IN [19]

\begin{tabular}{l||c|c|c|c|c|c|c||l|l|l|l}
\hline \hline & Poly & Rat & $\begin{array}{c}\text { Loc } \\
d=1\end{array}$ & $\begin{array}{l}\text { Loc } \\
d=2\end{array}$ & RBF & MLP & SVR & $\begin{array}{l}\text { SVM } \\
\text {-ARMA }\end{array}$ & $\begin{array}{l}\text { SVR } \\
\text {-ARMA } 2 K\end{array}$ & $\begin{array}{l}\text { SVM } \\
\text {-ARMA }\end{array}$ & $\begin{array}{l}\text { SVR } \\
\text {-ARMA }\end{array}$ \\
\hline \hline MG17 & -1.95 & -1.14 & -1.48 & -1.89 & -1.97 & -2.00 & -2.36 & -2.83 & -2.87 & -2.88 & -2.86 \\
\hline MG30 & -1.40 & -1.33 & -1.24 & -1.42 & -1.60 & -1.5 & $\mathbf{- 1 . 8 7}$ & -1.73 & -1.76 & -1.73 & -1.76 \\
\hline \hline
\end{tabular}

\section{EXPERIMENTAL RESULTS}

In this section, we compare the performance of the SVR and the SVM-ARMA formulations in the previous sections. In all kernel computations, we used the Gaussian kernel, which provides universal nonlinear mapping capabilities and computational convenience [17], [18]. Different types of kernels (linear, polynomial, etc.) could be considered for the input, output or cross-information kernels, according to a priori knowledge of the system.

Example 1. Nonlinear Feedback System: We frst consider the following system. Input DTP is generated with Lorenz equations, given by $d u / d t=-\rho u+\rho y, d y / d t=-u z+r u-y$, and $d z / d t=u y-b z$, and using $\rho=10, r=28$, and $b=8 / 3$. Only component $u$ is used as the input signal to the system, and it enters through an eighth-order low-pass FIR $\mathrm{f}$ lter $H(z)$ with cutoff frequency $\omega_{n}=0.5$ and normalized gain $6 \mathrm{~dB}$ at $\omega_{n}$. The output signal goes through a feedback loop consisting of a high-pass minimum-phase channel $G(z)=\left(1.00+2.01 z^{-1}+1.46 z^{-2}+0.39 z^{-3}\right)^{-1}$ and then distorted with nonlinearity $f(\cdot)=\log (\cdot)$.

This system was used to generate 10000 input-output DTP samples that were split into a training set (f rst 50 samples) and a test set (following 500 samples). The experiment was repeated 100 times with randomly selected starting points in the DTP. Free parameters were selected through eight-fold cross-validation using the training set, and average results for the test set are shown in Table I for mean error (ME), mean-squared error (MSE), mean-absolute error (MAE), correlation coeff cient $(r)$, and normalized MSE (nMSE $=\log _{10}(\sqrt{\mathrm{MSE} / \operatorname{var}(y)})$ of models in the test set. It is worth noting that SVM-ARMA ${ }_{2 K}$ is the best model for this example, and that also SVR-ARMA $A_{K}$ and SVM-ARMA $4 K$ outperform the SVR. However, the composite SVR-ARMA ${ }_{4}$ turns to be here the worst model specif cation. SVR-ARMA ${ }_{4}$ is a more complex model, which can be appropriate for much more complex dynamics; otherwise, the complexity of the model may degrade the generalization performance.

Example 2. The Mackey-Glass Time Series: We also compared the performance of SVM models in the standard Mackey-Glass time-series prediction problem following the same approach as in [20], where the use of the standard SVR was originally presented for time-series prediction. This classical high-dimensional chaotic system is generated by the following delay differential equation:

$$
\frac{d x(t)}{d t}=-0.1 x(t)+\frac{0.2 x\left(t-t_{\Delta}\right)}{1+x\left(t-t_{\Delta}\right)^{10}}
$$

with delays $t_{\Delta}=17$ and 30, yielding time series MG1 7 and MG30, respectively. For comparison with [20], we considered 500 training samples and used next 1000 for free parameter selection (validation set). This procedure also allows us a direct comparison with previous results in the literature [19], [21], [22], in terms of nonlinear MSE (nMSE).

Results are shown in Table II. The SVR algorithm outperformed the preceding methods for both time series. The methods proposed here widely outperform SVR in MG17; note that a difference of 0.5 between SVR and SVM-ARMA $4 K$ in nMSE is equivalent to almost one order of magnitude in MSE. Nevertheless, they did not outperform SVR in MG 30; this could be due to differences in the kernel choice (RBF instead of trigonometric kernel [20]) or the considered embedding.

\section{CONCLUSION}

This paper presents the explicit formulation of nonlinear SVM-ARMA models in RKHS, which makes possible to scrutinize the statistical properties and the time-series structure in system identif cation problems. In addition, a full family of methods for nonlinear system identif cation have been proposed, by taking advantage of composite kernels, in which dedicated mappings are used for input, output, and cross terms. Simulation results reveal the potential capabilities of this approach, as recently demonstrated in the f eld of image processing [23]. This framework also allows a successful integration and combination of nonlinear SVR and SVM-ARMA models.

\section{APPENDIX I}

\section{NONLINEAR SVM-ARMA AND PREDICTION ERROR METHODS}

Property 7: Let us denote quadratic cost conditions (QCC) as $\varepsilon=0$ and $C=\infty$ in (12). Then, for QCC we have that model weights in (19) are proportional to the residuals, that is $\beta_{n}=(1 / \gamma) e_{n}$. The proof is immediate by making $\varepsilon=0$ and $C=\infty$ in (10).

Property 8: For QCC, the linear SVM-ARMA model can be viewed as a regularization of the normal equations of the Wiener flter for system identif cation. See [11] for proof.

Theorem 1: For QCC, nonlinear SVM-ARMA ${ }_{2}$ algorithm solves the regularized normal equations in the RKHS.

Proof: Let $\phi_{u}\left(\boldsymbol{x}_{i}\right) \in \mathcal{H}_{u}$ and $\phi_{y}\left(\boldsymbol{y}_{i}\right) \in \mathcal{H}_{y}$, vectors of size $\left(H_{u} \times 1\right)$ and $\left(H_{y} \times 1\right)$, respectively, for $i=1, \ldots, N$. Let the data matrix in each RKHS be given by $\boldsymbol{\Phi}_{u}=\left[\boldsymbol{\phi}_{u}\left(\boldsymbol{u}_{1}\right), \ldots, \boldsymbol{\phi}_{u}\left(\boldsymbol{u}_{N}\right)\right]$ and $\boldsymbol{\Phi}_{y}=\left[\boldsymbol{\phi}_{y}\left(\boldsymbol{y}_{1}\right), \ldots, \boldsymbol{\phi}_{y}\left(\boldsymbol{y}_{N}\right)\right]$. Then, the matrix-form equation of the model for the observed data is $\boldsymbol{y}=\boldsymbol{\Phi}_{u}^{T} \boldsymbol{b}+\boldsymbol{\Phi}_{y}^{T} \boldsymbol{a}+\boldsymbol{e}$. Dual problem (18) can now be expressed (in matrix form) as the maximization of

$$
L_{\mathrm{QCC}}^{2 k}(\boldsymbol{e})=\frac{1}{2 \gamma^{2}} \boldsymbol{e}^{T}\left(\boldsymbol{\Phi}_{y}^{T} \boldsymbol{\Phi}_{y}+\boldsymbol{\Phi}_{u}^{T} \boldsymbol{\Phi}_{u}\right) \boldsymbol{e}-\frac{1}{\gamma} \boldsymbol{y}^{T} \boldsymbol{e}+\frac{1}{2 \gamma} \boldsymbol{e}^{T} \boldsymbol{e} .
$$

By making zero the gradient of $L_{\mathrm{QCC}}^{2 k}$ and after some manipulations, the following is obtained:

$$
\begin{array}{r}
\boldsymbol{\Phi}_{y}^{T} \boldsymbol{\Phi}_{y} \boldsymbol{y}-\boldsymbol{\Phi}_{y}^{T} \boldsymbol{\Phi}_{y} \boldsymbol{\Phi}_{u}^{T} \boldsymbol{b}-\boldsymbol{\Phi}_{y}^{T} \boldsymbol{\Phi}_{y} \boldsymbol{\Phi}_{y}^{T} \boldsymbol{a}+\boldsymbol{\Phi}_{u} \boldsymbol{\Phi}_{u}^{T} \boldsymbol{y}-\boldsymbol{\Phi}_{u}^{T} \boldsymbol{\Phi}_{u} \boldsymbol{\Phi}_{u}^{T} \boldsymbol{b}- \\
\boldsymbol{\Phi}_{u}^{T} \boldsymbol{\Phi}_{u} \boldsymbol{\Phi}_{y}^{T} \boldsymbol{a}-\gamma \boldsymbol{\Phi}_{u}^{T} \boldsymbol{b}-\gamma \boldsymbol{\Phi}_{y}^{T} \boldsymbol{a}=0 .
\end{array}
$$

By denoting $\boldsymbol{\Phi}_{y} \boldsymbol{\Phi}_{y}^{T}=\boldsymbol{R}_{y y}, \boldsymbol{\Phi}_{u} \boldsymbol{\Phi}_{u}^{T}=\boldsymbol{R}_{u u}, \boldsymbol{\Phi}_{u} \boldsymbol{\Phi}_{y}^{T}=\boldsymbol{R}_{u y}, \boldsymbol{\Phi}_{y} \boldsymbol{\Phi}_{u}^{T}=$ $\boldsymbol{R}_{y u}$, and

$$
\boldsymbol{r}_{z y}^{2 k}=\left[\begin{array}{c}
\boldsymbol{\Phi}_{y} \boldsymbol{y} \\
\boldsymbol{\Phi}_{u} \boldsymbol{y}
\end{array}\right] \quad \boldsymbol{R}^{2 k}=\left[\begin{array}{ll}
\boldsymbol{R}_{y y} & \boldsymbol{R}_{y u} \\
\boldsymbol{R}_{u y} & \boldsymbol{R}_{u u}
\end{array}\right]
$$

the following term grouping can be done:

$$
\left[\boldsymbol{\Phi}_{y}^{T}, \boldsymbol{\Phi}_{u}^{T}\right] \boldsymbol{r}_{z y}^{2 k}=\left[\boldsymbol{\Phi}_{y}^{T}, \boldsymbol{\Phi}_{u}^{T}\right]\left(\boldsymbol{R}^{2 k}+\gamma \boldsymbol{I}\right)\left[\boldsymbol{a}^{T}, \boldsymbol{b}^{T}\right]^{T}
$$

which holds if and only if $\boldsymbol{r}_{z y}^{2 k}=\left(\boldsymbol{R}^{2 k}+\gamma \boldsymbol{I}\right)\left[\boldsymbol{a}^{T}, \boldsymbol{b}^{T}\right]^{T}$, that are the 5 regularized Wiener equations in RKHS. 
Therefore, the SVM-ARMA $2 K$ formulation for nonlinear kernels and QCC leads naturally to the statement of the equations in the joint RKHS. Note that for some Mercer's kernels, the dimension of these equations can be inf nite; however, as far as we are not solving them explicitly, but rather implicitly in (18) and by means of the kernel trick, we will still be able to scrutinize the statistical properties of the time-series problem, specially if these properties can be conveniently expressed with dot products in the RKHS and subsequently analyzed using Mercer's kernels.

\section{APPENDIX II}

\section{NONLINEAR SVM-ARMA AND CORRELATION METHODS}

$\mathrm{CM}$ for system identif cation are based on the assumption that a good model produces residuals that are uncorrelated with past data, and, consequently, these methods minimize the cross correlation between a function of the residuals and a transformation of the data, both of them being possibly nonlinear. Different approaches in the literature [1] are based on different methods for determining suitable residual functions and data transformations.

Property 9: For QCC, the nonlinear SVM-ARMA ${ }_{2}$ system identif cation model minimizes the cross correlation between the data transformed to the RKHS and the residuals.

Proof: Taking into account (15) and Property 2, we have $\boldsymbol{a}=\boldsymbol{\Phi}_{y} \boldsymbol{e}$ and $\boldsymbol{b}=\boldsymbol{\Phi}_{u} \boldsymbol{e}$, and according to the primal problem statement (12), we are minimizing the $L_{2}$ norm of the coeff cients, even though they are in the RKHS and they cannot be explicitly known (see [11] for details on the equivalent property for linear SVM-ARMA).

Property 10: Under the set of nonzero and $\mathrm{f}$ nite possible values for the free parameters of the $\varepsilon$-Huber cost function $(0<\varepsilon, C, \gamma<\infty)$, the nonlinear SVM-ARMA $\mathrm{A}_{2}$ system identif cation algorithm minimizes the correlation between the data and a nonlinear transformation of the residuals.

Proof: For each given f xed subset of the free parameters $0<\varepsilon$, $C, \gamma<\infty$, the nonlinear relationship between the model coeff cients and the residuals is given by (10). According to (15) and (10), we have $\boldsymbol{a}=\boldsymbol{\Phi}_{y} g_{n l}(\boldsymbol{e})$ and $\boldsymbol{b}=\boldsymbol{\Phi}_{u} g_{n l}(\boldsymbol{e})$, which stand for the model coeff cients being the (uncoupled) cross correlation between the nonlinearly transformed residuals and the data in the RKHS. Furthermore, we are minimizing the norm of these coeff cients in (12).

\section{REFERENCES}

[1] L. Ljung, System Identification. Theory for the User, 2nd ed. Englewood Cliffs, NJ: Prentice-Hall, 1999.

[2] O. Nelles, Nonlinear System Identification. From classical approaches to Neural Networks and Fuzzy Models. New York: Springer-Verlag, 2001.

[3] V. Vapnik, Statistical Learning Theory (Adaptive and Learning Systems for Signal Processing, Communications, and Control). New York: Wiley, 1998.

[4] A. J. Smola and B. Schölkopf, A Tutorial on Support Vector Regression, Royal Holloway College, Univ. London, London, U.K., Tech. Rep. NC-TR-98-030, 1998.

[5] G. Camps-Valls, J. L. Rojo-Álvarez, and M. Martínez-Ramón, Eds., Kernel Methods in Bioengineering, Signal and Image Processing. Hershey, PA: Idea Group Inc., 2006.

[6] R. F. Drezet and P. M. L. Harrison, "Support vector machines for system identif cation," in UKACC Intl. Conf. Control '98, Swansea, U.K., Sep. 1998, vol. 1, pp. 688-692.

[7] A. Gretton, A. Doucet, R. Herbrich, P. J. W. Rayner, and B. Schölkopf, "Support vector regression for black-box system identif cation," in Proc. 11th IEEE Signal Process. Workshop Stat. Signal Process., 2001, pp. 341-344.

[8] J. A. K. Suykens, "Support vector machines: a nonlinear modelling and control perspective," Euro. J. Control, Special Issue on Fundamental Issues in Control, vol. 7, no. 2-3, pp. 311-327, Aug. 2001.
[9] I. Goethals, K. Pelckmans, J. A. K. Suykens, and B. De Moor, "Subspace identif cation of Hammerstein systems using least squares support vector machines," IEEE Trans. Autom. Control, vol. 50, no. 10, pp. 1509-1519, Oct. 2005.

[10] M. Espinoza, J. A. K. Suykens, and B. De Moor, "Kernel based partially linear models and nonlinear identif cation," IEEE Trans. Autom. Control, vol. 50, no. 10, pp. 1602-1606, Oct. 2005.

[11] J. L. Rojo-Álvarez, M. Martínez-Ramón, A. R. Figueiras-Vidal, M. dePrado Cumplido, and A. Artés-Rodríguez, "Support vector method for ARMA system identif cation," IEEE Trans. Signal Process., vol. 52, no. 1, pp. 155-64, Jan. 2004.

[12] J. L. Rojo-Álvarez, G. Camps-Valls, M. Martínez-Ramón, E. SoriaOlivas, A. Navia Vázquez, and A. R. Figueiras-Vidal, "Support vector machines framework for linear signal processing," Signal Process., vol. 85, no. 12, pp. 2316-2326, 2005.

[13] N. Cristianini and J. Shawe-Taylor, An Introduction to Support Vector Machines and Other Kernel-Based Learning Methods. Cambridge, U.K.: Cambridge Univ. Press, 2000.

[14] D. G. Luenberger, Linear and Nonlinear Programming. Reading, MA: Addison-Wesley, 1984.

[15] A. Papoulis, Probability Random Variables and Stochastic Processes, 3rd ed. New York: McGraw-Hill, 1991.

[16] M. C. Reed and B. Simon, Functional Analysis. New York: Academic Press, 1980, vol. I, Methods of Modern Mathematical Physics.

[17] B. Schölkopf and A. Smola, Learning With Kernels. Support Vector Machines, Regularization, Optimization and Beyond. Boston, MA: MIT Press, 2002.

[18] S. S. Keerthi and C.-J. Lin, "Asymptotic behaviors of support vector machines with Gaussian kernel," Neural Comput., vol. 15, no. 7, pp. 1667-1689, 2003.

[19] M. Casdagli and S. Eubank, "Nonlinear modeling and forecasting," in Proc. Santa Fe Inst. Studies in the Science of Complexity, Nov. 1992, vol. XII.

[20] S. Mukherjee, E. Osuna, and F. Girosi, "Nonlinear prediction of chaotic time series using a support vector machine," in Proc. IEEE Workshop Neural Netw. Signal Process. VII, J. Principe, L. Gile, N. Morgan, and E. Wilson, Eds., New York, 1997, pp. 511-520.

[21] A. Lapedes and R. Farber, Nonlinear Signal Processing Using Neural Networks: Prediction and System Modeling, Los Alamos Nat. Lab., Tech. Rep. LA-UR-87-2662, 1987.

[22] J. D. Farmer and J. J. Sidorowich, "Predicting chaotic time series," Phys. Rev. Lett., vol. 59, p. 543, 1987.

[23] G. Camps-Valls, L. Gomez-Chova, J. Muñoz-Marí, J. Vila-Francés, and J. Calpe-Maravilla, "Composite kernels for hyperspectral image classif cation," IEEE Geosci. Remote Sens. Lett., vol. 3, no. 1, pp. 93-97, Jan. 2006. 